\title{
Coping strategies observed in women with rheumatoid arthritis
}

\author{
Mariola Janiszewska ${ }^{1, B-D \oplus}$, Agnieszka Barańska ${ }^{1, A-D \oplus}{ }^{\text {, Krzysztof Kanecki' }}{ }^{2, A, D-F}{ }^{\oplus}$, Agata Karpińska ${ }^{1, E \oplus}$, \\ Ewelina Firlej, ${ }^{3, A-B, D \oplus}$, Magdalena Bogdan ${ }^{2, A, E-F} \oplus$ \\ ${ }^{1}$ Department of Informatics and Medical Statistics with E-learning Laboratory, Medical University, Lublin, Poland \\ 2 Department of Social Medicine and Public Health, Medical University, Warsaw, Poland \\ ${ }^{3}$ Cosmetology and Aesthetic Medicine Unit, Medical University, Lublin, Poland \\ A - Research concept and design, B - Collection and/or assembly of data, C - Data analysis and interpretation, \\ $D$ - Writing the article, E - Critical revision of the article, $F$ - Final approval of article
}

Janiszewska M, Barańska A, Kanecki K, Karpińska A, Firlej E, Bogdan M. Coping strategies observed in women with rheumatoid arthritis. Ann Agric Environ Med. 2020; 27(3): 401-406. doi: 10.26444/aaem/110958

\begin{abstract}
When faced with a chronic disease such as rheumatoid arthritis, the patient attempts to cope with the stressful situation by applying coping strategies. The main aim of rheumatoid treatment is not only improving health but also increasing the quality of life.

The research objective was to determine the relationship among socio-demographic factors, duration of the disease and its associated ailments, attitude to the disease, self-assessment of one's knowledge of RA, and the application of coping strategies in stressful situations by women with rheumatoid arthritis.

The study involved 193 patients of the Clinic of Rheumatology and Systemic Connective Tissue Diseases, and the Rheumatology Unit of the Specialist Outpatient Clinic of the Independent Public Teaching Hospital No. 4 in Lublin, from November 2016 - June 2017.

The Coping Orientations to Problems Experienced Inventory (COPE) Questionnaire and an author's Original Questionnaire were used in the study. Analysis of variance (ANOVA) and Tukey's range test were applied for statistical analysis. A p-value $<0.05$ defined the statistical differences. Analysis was performed using the commercial SPSS Statistics 19 software (IBM Corp., Armonk, NY, USA).

The respondents usually use instrumental social support (11.5 \pm 1.7$)$, focus on and vent emotions (11.4 \pm 1.9$)$, use emotional social support (11.4 \pm 1.8 ), employ active coping (11.4 \pm 2.1$)$ and positive reinterpretation and growth (11.2 \pm 1.8$)$, and least often

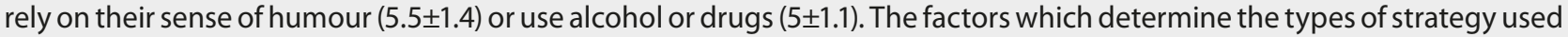
are age, education, the duration of the disease, ailments experienced, and attitude towards the disease. Those respondents who declared a greater knowledge of RA more often applied positive reinterpretation and growth, and more rarely used alcohol or drugs.

In the treatment and rehabilitation processes it is important to reinforce in the patient positive expectations for the treatment, seek advantages and benefits in one's present health status, and educate patients about the disease, its therapy and appropriate coping strategies.
\end{abstract}

\section{Key words}

rheumatoid arthritis, pain, psychological stress, coping strategies

\section{INTRODUCTION}

Rheumatoid arthritis (RA) is a chronic, autoimmune, systemic, connective-tissue disease characterised by progressive synovitis in symmetrical joints, which leads to severe disabilities and premature mortality $[1,2,3]$. The main symptoms which characterise RA include persistent pain, swelling, deformation of joints, morning stiffness and general tiredness. Due to constant pain and progressing disability, people affected by rheumatoid arthritis also may suffer from negative psychological consequences from the disease, such as mood changes, depression, and anxiety $[4,5]$.

The fact of contracting a chronic disease such as RA can be regarded as a critical, stressful, life event which requires the patient to adapt to the new circumstances [6]. The disease and associated psychological stress trigger a response which

Address for correspondence: Magdalena Bogdan, Department of Social Medicine and Public Health, Medical University of Warsaw, Poland

E-mail:mminko@onet.eu

Received: 17.05.2019; accepted: 21.07.2019; first published: 07.08.2019 takes the form of a coping strategy. Coping is defined as making cognitive or behavioural efforts to face internal and external requirements which impose a burden on, or exceed one's resources [7]. Four types of coping strategy can be distinguished. These are the cognitive approach and behavioural approach, which are problem-focused, and cognitive avoidance and behavioural avoidance, which are emotion-focused, on controlling one's emotional reaction to the danger [8]. Coping with difficult situations acts as a moderator between stress and mental well-being. Therefore, whether or not the effects of stress become exacerbated depends on the effectiveness of coping [9]. Coping can be active, and, for example, take the form of learning a new profession, or passive, e.g. turning to God through prayer. It can also assume an ongoing form, i.e. relating to the current situation associated with the disease, or a habitual one, which has always been used. In the case of rheumatoid arthritis, it is preferable that coping assumes the active and ongoing form, with emotion regulation also being an important factor $[10,11]$. 
To sum up, with a stressful condition such as a chronic disease, depending on the available internal and external resources, the patient develops certain mechanisms to cope with difficult circumstances. Applying appropriate coping strategies allows development of the right attitude to the disease, to undertake appropriate treatment and to adapt to one's new health status.

\section{OBJECTIVE}

The aim of the research was to determine the relationship among socio-demographic factors, duration of the disease and its associated ailments, attitude to the disease, selfassessment of one's knowledge of RA, and the application of coping strategies in stressful situations by women with rheumatoid arthritis.

\section{MATERIALS AND METHOD}

The study was conducted at the Clinic of Rheumatology and Systemic Connective Tissue Diseases, and the Rheumatology Unit of the Specialist Outpatient Clinic of the Independent Public Teaching Hospital No. 4 in Lublin, Eastern Poland, from November 2016 - June 2017. The study enrolled 193 women treated for rheumatoid arthritis (mean age $63 \pm 9$, range min-max age: $45-89$ years). Inclusion criteria:

- RA treatment;

- women aged 45 and over;

- being a resident of the Lublin Macroregion;

- giving consent to participate in the study.

Exclusion criteria:

- lack of consent to participate in the study.

Two research instruments were used:

1. Original author's survey questionnaire, in two parts. The first part comprised a personal information section with questions on the subject of the study - socio-demographic data. The second part featured open and closed questions aimed to provide information on the analysed area. Information on the duration of the disease and related ailments, the approach to the disease and self-assessment of knowledge about the disease was obtained by using the author's Questionnaire. The information provided was collected while completing questionnaires in an individual interview with each respondent. The author's Questionnaire was created and used for collecting information not included in the COPE Questionnaire. It was not a part of comparison analysis.

2. The Coping Orientations to Problems Experienced Inventory (COPE) (C. S. Carver, M. F. Scheier, J. K. Weintraub, 1989) in the Polish adaptation (Z. Juczyński, N., Ogińska-Bulik) [12] was used to study the coping strategies in stressful situations.

The duration of the RA treatment, ailments experienced, attitudes to the disease, and the self-assessment of knowledge of the disease, were determined using an Original Questionnaire.

The COPE Inventory makes it possible to assess 15 coping strategies. It is composed of 60 items to be answered on a 4-point scale, from 1 - "I usually don't do this at all" to $4=$ "I usually do this a lot". Three scales were distinguished in the COPE Inventory: "active coping", "seeking support and focusing on emotions", and "avoidance behaviours". The "active coping" scale is composed of 5 strategies: planning, positive reinterpretation and growth, active coping, suppression of competing activities, and restraint. The "seeking support and focusing on emotions" scale is composed of 4 strategies: seeking emotional social support, focus on and venting emotions, seeking out instrumental social support, and turning to religion. The "avoidance behaviours" scale is composed of 6 strategies: denial, behavioural disengagement, humour, mental disengagement, alcohol and drug use, and acceptance. The values of Cronbach's alpha for the individual scales range from 0.48-0.94, and are the lowest for "mental disengagement" and "active coping", and the highest for "turning to religion" [12].

Ethical issues. The study was conducted in accordance with the principles for human research set by the Declaration of Helsinki. The study was voluntary, and each participant signed a consent form and was assured that the study would be kept anonymous. The purpose of the study and how the questionnaires should be completed was explained to the respondents. Questionnaires were completed by the respondents in the presence of an interviewer. In case of difficulties, the interviewer assisted in completing the questionnaires.

Statistical analysis. Continuous data were reported as means \pm SD and the minimum-maximum range. Before using parametric tests, assumptions about the normality of distributions were checked using the Shapiro-Wilk test. An analysis of variance (ANOVA) was applied in the statistical analysis to check whether there were statistical differences in terms of ratio variables among more than 2 groups. In order to determine which groups differred significantly from one another, Tukey's range test was carried out. The means for groups with significant differences were highlighted. Spearman's rho was applied to evaluate whether there were any statistically significant correlations between the rank variables and ratio variables. A p-value of $<0.05$ defined the statistical significance of the differences. The analysis was performed using commercial SPSS Statistics 19 software (IBM Corp., Armonk, NY, USA).

\section{RESULTS}

The study enrolled - 193 women treated for rheumatoid arthritis (mean age $63 \pm 9$, range min-max age: $45-89$ years). More than half the respondents (54.2\%) lived in urban areas. The majority of respondents $(62.5 \%)$ were in a relationship (marriage or partnership), $19.2 \%$ were widowed, $11.4 \%$ were divorcees, and $6.7 \%$ were single. Most of the study participants had vocational education (35.4\%) and higher education (33.9\%), while other women attained secondary $(29.2 \%)$ or primary (1.6\%) education. Most of the patients (78.8\%) assessed their social-living conditions as good or very good, while the remaining $13.9 \%$ assessed them as bad or very bad.

The vast majority of the respondents $(81.3 \%)$ had been treated for rheumatoid arthritis for more than a year, 
and nearly half the studied women (42.5\%) for 1-5 years. Almost half of the respondents (46.4\%) experienced ailments associated with the disease every day, while $28.6 \%$ did so several times a week, and 25\% several times a month. Most respondents reported morning stiffness $(81.8 \%)$ and joint swelling, and $43.9 \%$ about bothersome joint pain. The pain limited the respondents' ability to perform everyday activities to a medium (54.2\% of the studied women) and a very large extent (14.6\% of the respondents). Regarding the fact of contracting RA, approximately $60 \%$ of all the respondents had come to terms with the disease, but nevertheless had not accepted it; only $24.4 \%$ had come to terms with the disease and had fully accepted it. Approximately $16 \%$ of the respondents stated that to-date they had not come to terms with the disease. When asked about their knowledge of RA, the respondents assessed it as good (68.8\%), very good (9.4\%) or limited (27.1\%); $8.2 \%$ of the respondents stated that they had no knowledge of this topic.

The stress associated with chronic disease results in the patient's attempting to cope with the existing, usually difficult, situation, and the way one responds to the stressful situation determines the types of the applied strategy. Coping strategies are specific efforts of a behavioural and psychological nature used to control, accept, eliminate, or minimise the effects of stressful events [13]. Table 1 presents a selection of the coping strategies applied by the studied women being treated for rheumatoid arthritis.

Table 1. COPE results for all respondents

\begin{tabular}{lc}
\hline COPE & \\
\hline Use of instrumental social support & $11.5 \pm 1.7(4-16)$ \\
\hline Focus on and venting emotions & $11.4 \pm 1.9(7-16)$ \\
\hline Use of emotional social support & $11.4 \pm 1.8(5-16)$ \\
\hline Active coping & $11.4 \pm 2.1(6-16)$ \\
\hline Positive reinterpretation and growth & $11.2 \pm 1.8(7-16)$ \\
\hline Turning to religion & $9.9 \pm 2(4-16)$ \\
\hline Planning & $9.3 \pm 2.2(4-16)$ \\
\hline Restraint & $8.5 \pm 1.8(5-14)$ \\
\hline Suppression of competing activities & $8.3 \pm 1.8(4-15)$ \\
\hline Denial & $7.7 \pm 1.3(4-12)$ \\
\hline Mental disengagement & $7.6 \pm 1.6(4-14)$ \\
\hline Acceptance & $7.6 \pm 1.8(4-16)$ \\
\hline Behavioural disengagement & $7.2 \pm 1.4(4-11)$ \\
\hline Humour & $5.5 \pm 1.4(4-12)$ \\
\hline Alcohol and drug use & $5 \pm 1.1(4-10)$ \\
\hline
\end{tabular}

Data are means $\pm S D$ (min-max range).

Statistical analysis of the mean results showed that of all the coping strategies, respondents most often indicated and used 5, i.e. using instrumental social support, focusing on and venting emotions, using emotional social support, active coping, and positive reinterpretation and growth. Other strategies were employed much more rarely, with avoidance strategies' being regarded as a risk factor associated with undesirable reactions to stress, such as humour and alcohol and drug use, being pursued the least often (Tab. 1).

It turned out that the older the respondent, the more often she employed such strategies as active coping $(\mathrm{p}<0.001)$, planning $(\mathrm{p}<0.005)$, using emotional social support $(\mathrm{p}<0.016)$, turning to religion $(\mathrm{p}<0.001)$, and humour $(\mathrm{p}<0.005)$, and less often, using mental disengagement $(\mathrm{p}<0.001)$. The correlation was observed between age and mental disengagement (rho=$0.310 ; \mathrm{p}<0.001$ ) and was negative, which means that as the respondents grew older, they were less inclined to mentally disengage from the disease.

Summing up, older patients tend to apply strategies encompassed within the "active coping" and "seeking support and focusing on emotions" scales than "avoidance behaviour".

Higher education fostered active coping in the studied individuals. The surveys show that the higher the level of declared education, the more often the respondents applied positive reinterpretation and growth $(\mathrm{p}<0.030)$, planning $(\mathrm{p}<0.037)$ and suppression of competing activities $(\mathrm{p}<0.037)$.

In general, hardly any statistically significant correlations were observed between the social conditions of the respondents and the coping strategies they employed. Social conditions correlated only with the use of emotional social support $(\mathrm{p}<0.020)$; therefore, the better the social conditions, the more frequent the search for emotional social support.

The passage of time from the onset of RA correlated significantly with a number of coping strategies. The longer the duration of the disease, the more often positive reinterpretation and growth $(\mathrm{p}<0.001)$, focus on and venting of emotions $(\mathrm{p}<0.031)$, and mental disengagement $(\mathrm{p}<0.001)$, were applied. Unfortunately, the respondents more rarely used active coping $(\mathrm{p}<0.008)$ or planning $(\mathrm{p}<0.035)$, but they also more rarely resorted to alcohol and drug use $(p<0.000)$. The majority of these correlations were weak. Only the correlations between the duration of the disease and the strategy consisting of alcohol and drug use (negative correlation) (rho=- 0.411; $\mathrm{p}<0.001)$ and the duration of the disease and positive reinterpretation and growth (positive correlation) (rho $=0.331, \mathrm{p}<0.001$ ), can be described as moderately strong. Therefore, in this case, the longer the duration of the disease, the more the respondents were inclined to apply positive reinterpretation and growth, and less prone to use avoidance strategies, such as alcohol and drug use.

The attitude to contracting rheumatoid arthritis significantly differentiates a number of coping strategies in difficult, disease-related, situations. Significantly higher means of active coping $(\mathrm{p}<0.001)$, positive reinterpretation and growth $(\mathrm{p}=<0.001)$, and planning $(\mathrm{p}<0.001)$, were recorded for those women who had come to terms with the disease and accepted, compared with the remaining respondents. On the other hand, higher scores for the use of emotional social support $(\mathrm{p}<0.012)$ were recorded among those respondents who had come to terms with the disease but had not accepted it, compared to those who had declared coming to terms with the disease and its acceptance. When compared with the other studied women, significantly lower results in the context of turning to religion $(\mathrm{p}<0.015)$, acceptance $(\mathrm{p}<0.001)$, and humour $(\mathrm{p}<0.019)$, characterised those respondents who had not come to terms with the disease, compared with the rest of the group. Significantly higher results in terms of focussing on and venting emotions $(p<0.000)$ and mental disengagement $(\mathrm{p}<0.000)$ were recorded in those respondents who had not come to terms with the disease than in the other women. Those respondents who had come to terms with the disease but had not accepted it resorted to alcohol and drug use significantly more frequently than respondents who had come to terms with the disease and had accepted it $(p<0.010)$. 
It turned out that the more often the respondents experienced ailments related to RA, the more they focused not only on active coping and seeking support and focusing on emotions through active coping $(\mathrm{p}<1.000)$, planning $(\mathrm{p}<0.001)$, the use of emotional social support $(\mathrm{p}<0.001)$, suppression of competing activities $(\mathrm{p}<0.010)$, and turning to religion $(\mathrm{p}<0.002)$, but also employed avoidance strategies, such as alcohol and drug use $(\mathrm{p}<0.001)$, humour, $(\mathrm{p}<0.001)$, and acceptance $(\mathrm{p}<0.001)$. Also, they were less inclined to use positive reinterpretation and growth $(\mathrm{p}<0.001)$, restraint, $(\mathrm{p}<0.038)$, denial $(\mathrm{p}<0.047)$, and mental disengagement $(p<0.001)$. The strongest of these correlations were moderately strong and observed between the frequency of experienced symptoms and planning ( $r h o=0.499, \mathrm{p}<0.001)$, and alcohol and drug use ( $r h o=0.494 ; \mathrm{p}<0.001)$; therefore, the more often the respondents experienced the ailments, the more often they planned and used alcohol or drugs.

Statistical analysis demonstrated that the more the physical pain related to the disease limited the respondents in everyday activities, the more often they apply active coping $(\mathrm{p}<0.001)$, planning $(\mathrm{p}<0.001)$, use of emotional social support $(\mathrm{p}<0.001)$, suppression of competing activities $(\mathrm{p}<0.001)$, turning to religion $(\mathrm{p}<0.005)$, focusing on and venting emotions $(p<0.048)$, as well as resorting to avoidance behaviours, such as alcohol and drug use $(\mathrm{p}<0.001)$ and humour $(p<0.001)$, the less often they become mentally disengaged $(p<0.001)$. The strongest of the aforementioned correlations was observed between the degree to which physical pain limited the respondents in everyday activities and planning $(\mathrm{rho}=0.491 ; \mathrm{p}<0.001)$.

Statistical analysis also revealed a correlation between selfassessment of the knowledge of rheumatoid arthritis and the applied strategies. The women who assessed their knowledge of the disease as higher often applied positive reinterpretation and growth $(\mathrm{p}<0.001)$, and more rarely resorted to alcohol and drug use $(\mathrm{p}<0.001)$. The strongest correlations were observed between self-assessment of knowledge and positive reinterpretation and growth $(\mathrm{rho}=0.301 ; \mathrm{p}<0.001)$.

\section{DISCUSSION}

The authors analyzed the types of coping strategies chosen by women suffering from rheumatoid arthritis. Building proper attitudes to cope with stressful situations among these patients is an important element of the comprehensive treatment of these patients, including improvement of the health-related quality of life. However, in the medical literature, this topic is not widely analyzed. The cognitive aim of this study was to describe a phenomenon not yet described in the professional literature: to fill this gap and open further possibilities for investigating the subject and issues arising. Among the coping strategies, there are active strategies, emotion-focused strategies, and avoidance strategies. Active coping strategies, which are the most appropriate in a stressful situation, are either behavioural or psychological reactions designed to change the nature of the stressor or the way it is perceived. Emotion-based strategies are employed as a response to anxiety about one's future, and the reactions of family and friends to the problematic situation and their readiness to extend support. Such strategies are used by those people who, in the face of stress, mainly focus on their own feelings and emotional experiences, and prefer wishful thinking and fantasising to rational action. On the other hand, avoidance strategies, characteristic of those individuals who tend to evade thinking about the problem, lead to undesirable behaviours, such as alcohol abuse or to emotional states such as withdrawal, which make it more difficult or impossible to react directly to stressful events [14].

The results of the authors' original studies demonstrate that the respondents affected by RA are much more inclined to apply emotion-focused strategies and those consisting of coping with stress and the difficult situation - the disease which affects them. The respondents seek instrumental and emotional social support, focus on emotions and venting them, try to actively cope with the problem, make positive reinterpretations, and are oriented towards growth. The use of undesirable avoidance strategies, such as underestimating the problem through humour or alcohol and drug abuse instead of implementing constructive solutions, was observed much more rarely. Similar results have been published by Berner et al. whose research focused on strategies for coping with illnesses, demographic and clinical factors associated with medication adherence among patients with RA. Respondents in the labove-mentioned research affected most frequently coping strategies such as active coping, diversion, and selfencouragement [15].

While active problem-focused coping is regarded as the optimal way of confronting stress, in the literature there are also reports which state that the emotion-focused strategies employed by women with chronic diseases result in discomfort caused by the stressful situation's becoming weaker [16, 17] and the regulation of emotional states [18]. However, most studies indicate a negative impact of the emotion-focused coping style on human functioning [19, 20]. For example, Kneebone and Martin [21] argue that the application of emotion-focused strategies and avoidance can result in adaptation difficulties. Holahan [22], in a similar fashion, regards avoidance behaviour as a psychological risk factor involving unfavourable reactions to stressful life events. Summing up, achieving a positive result when confronting difficult situations and maintaining biopsychosocial wellbeing is connected with the selection of the appropriate strategy [23].

Rheumatoid arthritis involves ailments which are hard to come to terms with. These include chronic pain, swelling and deformation of the joints, morning stiffness, tiredness, and even depression. The authors' original studies indicate that individuals who have come to terms with the disease and accepted the associated ailments are more inclined to apply positive strategies found within the "active coping" scale, and therefore use active coping, positive reinterpretation, and growth and planning. Such individuals are less prone to using alcohol or drugs, compared to those who cannot accept the disease despite coming to terms with it. McCracken et al. noted a relationship between accepting the disease and pain and improved physical and mental performance [24].

The authors of the current study also claim that the frequency of symptoms, in particular chronic pain, impacts on the types of coping strategies applied. Despite the fact that the more the ailments are experienced, the more often the respondents focus on active coping strategies, such as planning, the use of emotional social support, and turning to religion; they also use most of the avoidance strategies. It is of concern that one of the strongest positive correlations was observed between the frequency of symptoms and alcohol 
and drug use. According to Brown \& Nicassio, problemsolving, active coping, and controlling emotions can be regarded as opportunities to reduce pain, depression, and improve general functioning. On the other hand, passive coping, consisting of avoidance and escape, is correlated with greater depression, pain, and functional impairment [25].

Pain is not only the main symptom, but also a crucial stressor in RA patients. The chronic pain associated with RA is regarded as an uncontrollable stressor [4], which means that self-care options are not very effective when it comes to determining its course and impact [26]. Since one's coping approach in an uncontrollable context can only exacerbate the risk of maladjustment and frustration focusing on and coping with emotions and not problems, can be more beneficial in people affected by rheumatoid arthritis [27]. However, studies on cognitive and behavioural strategies intended to reduce the unpleasant feelings related to RA do not offer clear conclusions [4]. Only a few studies confirmed the adaptation role of such behaviour as emotional expression [28], or attempting to distance oneself from pain [29], while other studies did not confirm this [4]. For example, Ramirez-Maestre et al. [30], who studied chronic pain, demonstrated interrelations between active coping being pursued by patients and their better everyday functioning and reduced pain intensity. The results of the original study indicate that the more the physical pain associated with the disease, limited the studied women in their everyday activities, the more they declared the use of active coping strategies, involving in particular planning, seeking support, and focusing on emotions, as well as avoiding the problem through joking about the disease and using alcohol and drugs, which is unfortunate.

Furthermore, it is worth noting that, with age, patients tend to apply strategies encompassed within the "active coping" and "seeking support and focusing on emotions" scales more than "avoidance behaviours". Older women are less likely to mentally disengage from the problems related to falling ill.

In the original study, higher education fosters active coping strategies, befitting the disease, such as positive reinterpretation and growth, and planning and suppression of competing activities. Similarly, Parker et al. [31] indicate that a low level of attained education is associated with being emotion-focused and negative, evasive strategies in patients with ankylosing spondylitis. The social conditions of the studied women do not, to a significant extent, correlate with the coping strategies employed.

The length of time from the onset of RA correlates significantly with the number of coping strategies. If the diagnosis were made a long time in the past, this facilitates positive reinterpretation and growth, and focusing on emotional experiences, and also, unfortunately, mental disengagement. The longer the period from the time of diagnosis, the less often active coping and planning are applied. It is encouraging, however, that a longer duration of the disease is not conducive to alcohol and drug use.

The authors would also point to the fact that sufficient knowledge of RA, as declared by the respondents, is often associated with the implementation of a beneficial strategy consisting of positive reinterpretation and growth. The women who assessed their knowledge as higher are also less likely to employ avoidance behaviour, such as alcohol or drug abuse. Therefore, people who have reliable information about their health adopt attitudes fostering treatment.
In Poland, the number of RA sufferers is increasing [32]. People with rheumatoid arthritis experience stress caused by their disability and persistent pain and are dependent on other people. The exacerbation of stress is related to not utilising the appropriate active coping strategies [33], which is why treating, and rehabilitating RA patients should also see the addition of psychological interventions, psychotherapy, and education on the ways of coping with stressful situations.

Special attention should be paid to those RA patients who exhibit negative expectations determining the presence of strong, persistent pain, irrespective of any adopted coping measures. In such a case, an effective strategy may involve amplifying positive expectations towards medical treatment and identifying favourable aspects of one's current health status. Research suggests that clinical practice should take account of an optimistic attitude and the employed paincoping strategies.

\section{CONCLUSIONS}

1. The coping strategies most often applied by the studied women were: instrumental social support, focus on and venting emotions, use of emotional social support, active coping, and positive reinterpretation and growth. Humour and the use of alcohol or drugs were applied the least often.

2. Age, education, the duration of the disease, ailments experienced, and attitudes towards the disease, all affect the types of strategies used which can serve a preventive role, or have a negative impact on the treatment process.

3. Those respondents who declared greater knowledge of RA more often apply positive reinterpretation and growth, and more rarely used alcohol or drugs.

4. In the treatment and rehabilitation processes it is important to reinforce in the patient positive expectations for the treatment, seek advantages and benefits in one's present health status, and to educate patients about the disease, its therapy and the appropriate coping strategies.

Limitations and strengths of the study. A limitations is the small number of respondents and the limited area of the study. Therefore, there is a need for research involving a greater number of women from all over Poland. The strength of the study is the fact that it was performed in the area of a very important and current topic of using remedial strategies by women suffering from rheumatoid arthritis. In the face of an aging population and an increase in the number of chronically ill people, it is important to search for and build appropriate attitudes to cope with the disease by people of a stagnant age. This can be crucial for improving the health of patients with rheumatoid arthritis. The research was conducted in form of pilot study and continuation based on a wide scope of patients and using more specific statistical analysis is needed.

\section{REFERENCES}

1. Aletaha D, Neogi T, Silman A J, et al. References 2010 Rheumatoid arthritis classification criteria: An American College of Rheumatology/ European League Against Rheumatism collaborative initiative. Arthritis Rheum. 2010; 62(9): 2569-2581.

2. Wolfe F. The natural history of rheumatoid arthritis. J Rheumatol Suppl. 1996; 44: 13-22. 
3. Isomaki H. Long-term outcome of rheumatoid arthritis. Scand J Rheumatol Suppl. 1992; 95: 3-8.

4. Newth S, Delongis A. Individual differences, mood, and coping with chronic pain in rheumatoid arthritis: a daily process analysis. Psychol Health 2004; 19: 283-305.

5. Pincus T, Griffith J, Pearce S, et al. Prevalence of selfreported depression in patients with rheumatoid arthritis. Br J Rheumatol. 1996; 35: 879_ 883.

6. Poh LW, He HG, Chan WCS, et al. Experiences of Patients with Rheumatoid Arthritis: A Qualitative Study. Clin Nurs Res. 2017; 26(3): 373-380.

7. Lazarus RS, Folkman S. Stress, appraisal and coping. Springer, New York; 1984.

8. Lazarus R. Coping theory and research: past, present and future. Psychosom Med. 1993; 55: 234-247.

9. Folkman S, Lazarus RS. If it changes it must be aprocess: Study of emotion and coping during three stages of a college examination. J Pers Soc Psychol. 1985; 48: 150-170.

10. Günther V, Mur E, Kurz M, et al. Stable pattern of stress coping in patients with rheumatoid arthritis. Clin Exp Rheumatol. 1994; 12: $35-43$.

11. Van Middendorp H, Geenen R, Sorbi MJ, et al Emotion regulation predicts change of perceived health in patients with rheumatoid arthritis. Ann Rheum Dis. 2005; 64: 1071-1074.

12. Carver C S, Scheier M F, Weintraub J K. Assesing coping strategies: A theoretically based approach. J Pers Soc Psychol. 1989; 56: 267-283.

13. Watson MJ, Logan HL, Tomar SL. The influence of active coping and perceived stress on health disparities in a multi-ethnic low income sample. BMC Public Health 2008; 8: 41.

14. Krohne HW. Vigilance and cognitive avoidance as concepts in coping research. In: Krohne HW, editor. Attention and avoidance: Strategies in coping with aversiveness. Seattle (WA): Hogrefe \& Huber Publishers: 1993: $19-50$.

15. Berner C, Erlacher L, Fenzl KH, Dorner TE. Medication Adherence and Coping Strategies in Patients with Rheumatoid Arthritis: A CrossSectional Study. Int J Rheumatol. 2019: 1-8.

16. Suominen S, Helenius H, Blomberg H, at al. Sense of coherence as a predictor of subjective state of health, results of 4 years of follow-up of adults. J Psychosom Res. 2001; 50: 77-86.

17. Kim H S, Yeom H A, Seo Y S, at al. Stress and coping strategies of patientswith cancer. Cancer Nurs. 2002; 25(6): 425-31.

18. Vitaliano PP Russo J, Young HM, et al. Predictors of burden in spouse caregivers of individuals with Alzheimer's disease. Psychol Aging. 1991; 6: 392-402.
19. Haley WE, Roth DL, Coleton MI, at al. Appraisal, coping, and social support as mediators of well-being in black and white family caregivers of patients with Alzheimer's disease. J Consult Clin Psychol. 1996; 64: 121-129.

20. Almberg B, Grafström M, Winblad B. Major strain and coping strategies as reported by family members who care for aged demented relatives. J Adv Nurs. 1997; 26 (4): 683-91.

21. Kneebone I, Martin P.R Coping and caregivers of people with dementia. Br J Health Psychol. 2003; 8: 1-17.

22. Holahan CJ, Moos RH. Risk, resistance, and psychological distress: A longitudinal analysis with adults and children. J Abnorm Psychol. 1987; 96 (1): 3-13.

23. Hamarat E, Thompson D, Zabrucky KM, at al. Perceived Stress and Coping Resource Availability as Predictors of Life Satisfaction in Young, Middle-Aged, and Older Adults. Exp Aging Res. 2001; 27: 181-196.

24. McCracken L, Vowles K, Eccleston Ch. Acceptance of chronic pain: component analysis and a revised assessment method. Pain 2004; 107: 159-166.

25. Brown GK, Nicassio PM. Development of a questionnaire for the assessment of active and passive coping strategies in chronic pain patients. Pain 1987; 31: 53-64.

26. Fournier M, De Ridder D, Bensing J. Optimism and adaptation to chronic disease: The role of optimism in relation to self-care options of type 1 diabetes mellitus, rheumatoid arthritis and multiple sclerosis. Br J Health Psychol. 2002; 7: 409-432.

27. Affleck G, Pfeiffer C, Tennen H, et al. Attributional processes in rheumatoid arthritis patients. Arthritis Rheum. 1987; 30: 927-931.

28. Kerns RD, Rosenberg R, Jacob MC. Anger expression and chronic pain. J Behav Med. 1994; 17: 57-67.

29. Watkins KW, Shifren K, Park DC, et al. Age, pain, and coping with rheumatoid arthritis. Pain 1999; 82: 217-228.

30. Ramirez-Maestre C, Esteve R, Lopez A. Cognitive appraisal andcoping in chronic pain patients. Eur J Pain 2008; 12: 749-756.

31. Parker JC, Smarr KL, Hewett JE, et al. Health status, cognitive coping, and depressive symptoms: testing for a mediator effect. J Rheum. 2005; 32: $1584-1588$.

32. Iltchev P, Śliwczyński A, Czeleko T et al. Epidemiology of Rheumatoid Arthritis (RA) in rural and urban areas of Poland - 2008-2012. Ann Agric Environ Med. 2016; 23(2): 350-356.

33. Treharne GJ, Lyons AC, Booth DA et al. Psychological well-being across 1 year with rheumatoid arthritis: coping resources as buffers of percived stress. B J Health Psychol. 2007; 12(3): 323-345. 